
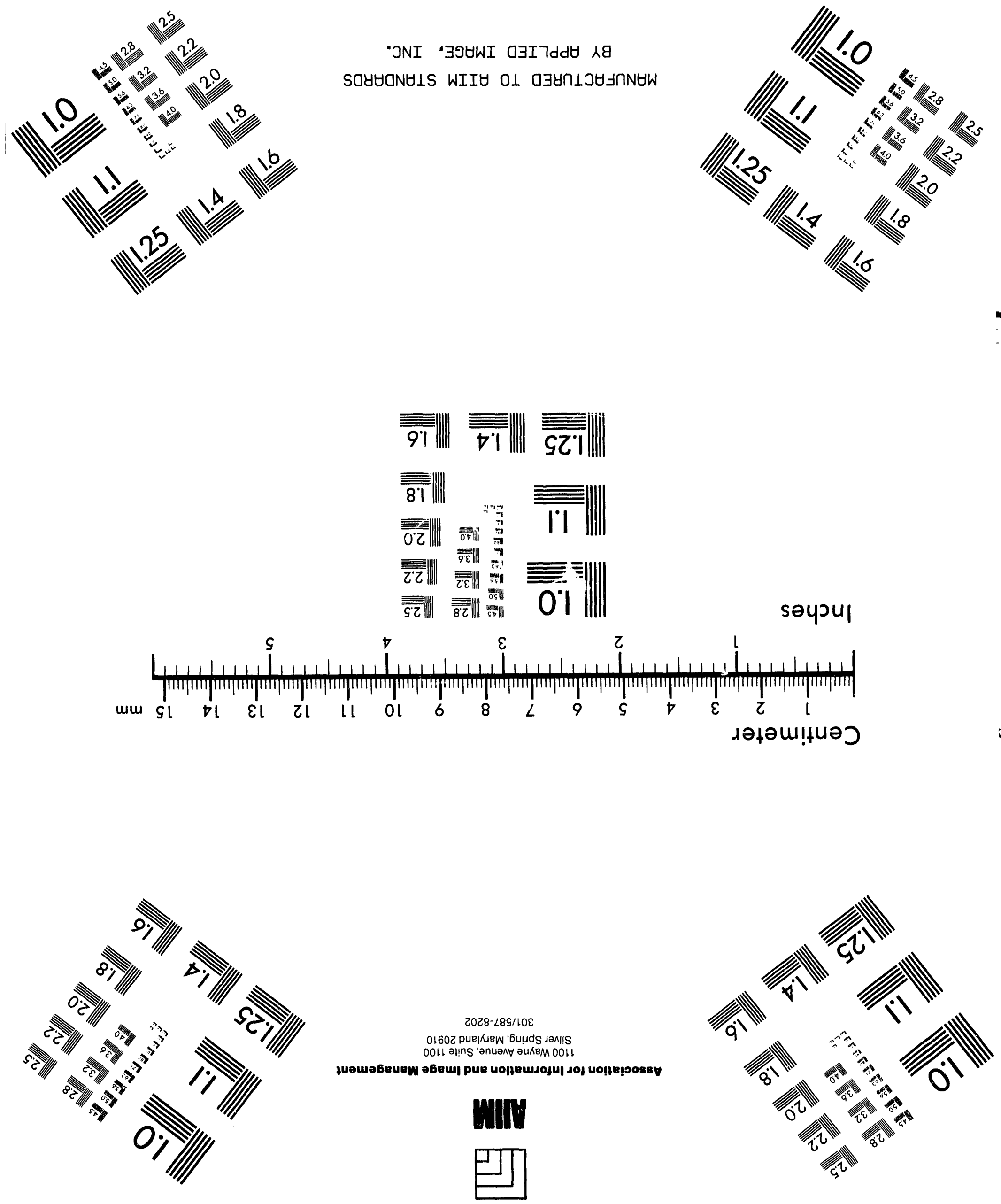

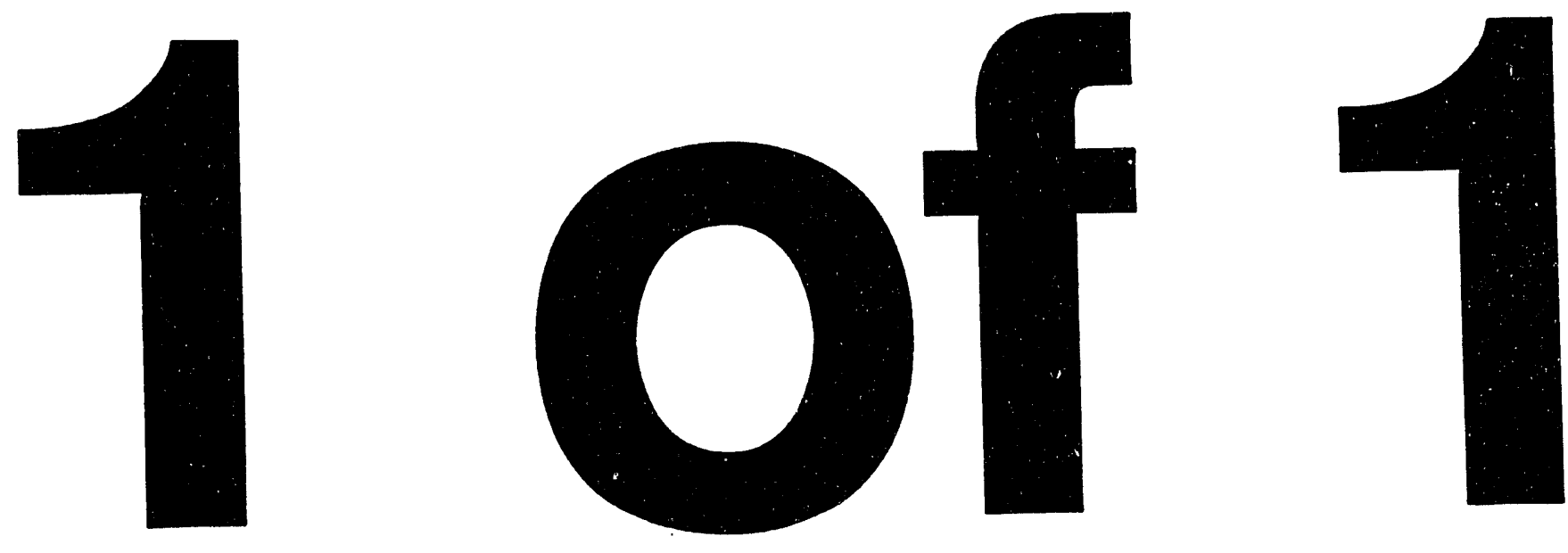


\title{
DETECTION EFFICIENCY AND DARK PULSE RATE OF ROCKWELL (SSPM) SINGLE PHOTON COUNTERS ${ }^{1}$
}

\author{
P.H. Eberhard and P.G. Kwiat \\ Physics Group, Lawrence Berkeley Laboratory \\ Berkeley, California 94720. U.S.A. \\ and \\ M.D. Petroff, M.G. Stapelbroek and H.H. Hogue \\ Rockwell International Corporation Science Cienter \\ 3370 Miraloma Ave., Anaheim, CA 9280:3
}

\begin{abstract}
This paper describes direct photon counting data on several pixels of the Rockwell SSPM detector array, about which absolute single photon detection efficiencies and time responses were reported previously by $K$ wiat et al. (ref 1,2$)$. The new data shows a substantially larger efficiency, $85 \%$, and suggests that damage in the optical fiber employed in the earlier data was responsible for the lower number, $70 \%$. When the results of this paper are analyzed using reasonable assumptions, one concludes that several improvements of the SSPM detector are still possible to bring the photon counting efficiency close to or larger than $95 \%$.
\end{abstract}

\section{INTRODUCTION}

A series of measurements of the absolute efficiency and time response of two different types of single-photon detectors was performed at Berkeley in August 1992 (ref. 1,2). The detectors were chosen because of their reported high quantum efficiencies and speed of response. One of these consisted of an array of solid-state photo-multipliers (SSPMs) from Rockwell (ref. 3) and the the other a pair of Geiger mode avalanche photodiodes from EG\&G (ref. 4). The SSPMs and their later versions, referred to by Rockwell as Visible light photon counters (VLPCs) (ref. 5,6) utilize the same impurity band avalanche effect in silicon at liquid helium temperature. These types of detectors were known to have a quantum efficiency of better than $60 \%$ and response time shorter than 10 us. The Berkeley absolute measurement provided a minimum detection efficiency value of $70.9 \%$ at a wavelength of $633 \mathrm{~nm}$. However, subsequent tests on the SSPMs suggested a possible degradation of transmission in the input fibers. The measurements reported here used the same SSPM chip. but equipped with improved fibers, less subject (though not quite immune) to degradation of transmission. These measurements allow a re-evaluation of the achievable SSPM or VLPC detection efficiencies. The subject of high photon detection efficiency is important for quantum cryptography and loophole-frec tests of Bell's inequalities.

\footnotetext{
${ }^{3}$ This work was supported by the Director, Office of Energy Research, Office of High Energy and Nuclear Physics, Division of High Energy Physics of the U.S. Department of Energy under Contract DE-AC03. $76 \mathrm{SF} 00098$
} 


\section{MEASUREMENTS}

Three SSPM detectors on an 8-pixel chip were available for testing. Each of the three pixels was connected to a different optical fiber to bring light from an optical arrangement at room temperature to the device operating at a temperature of $6 \mathrm{~K}$. Each of them came with its output comnected to a separate charge integrator at room temperature. The three devices were studied in various conditions. In almost all the tests, the wavelength was selected to be $660 \mathrm{~nm}$ by a filter of $10 \mathrm{~nm}$ FWHM. Data on efficiencies and background count rate were recorded. Typical signal pulse heights were about $.7 \mathrm{mV}$ al the output of the charge integrator.

In the first test setup, no additional optical feature was added to the assembly as it came from Rockwell International Corporation. Efficiencies were measured and found to fall in a range from 70 to $76 \%$, depending on the device, on the discriminator level, on device bias voltage, and on the temperature. A curve of efficiency versus discriminator level is shown on Fig. 1. The horizontal scale on Fig. 1 indicates the pulse height at the output of the charge integrator that triggers the discriminator.

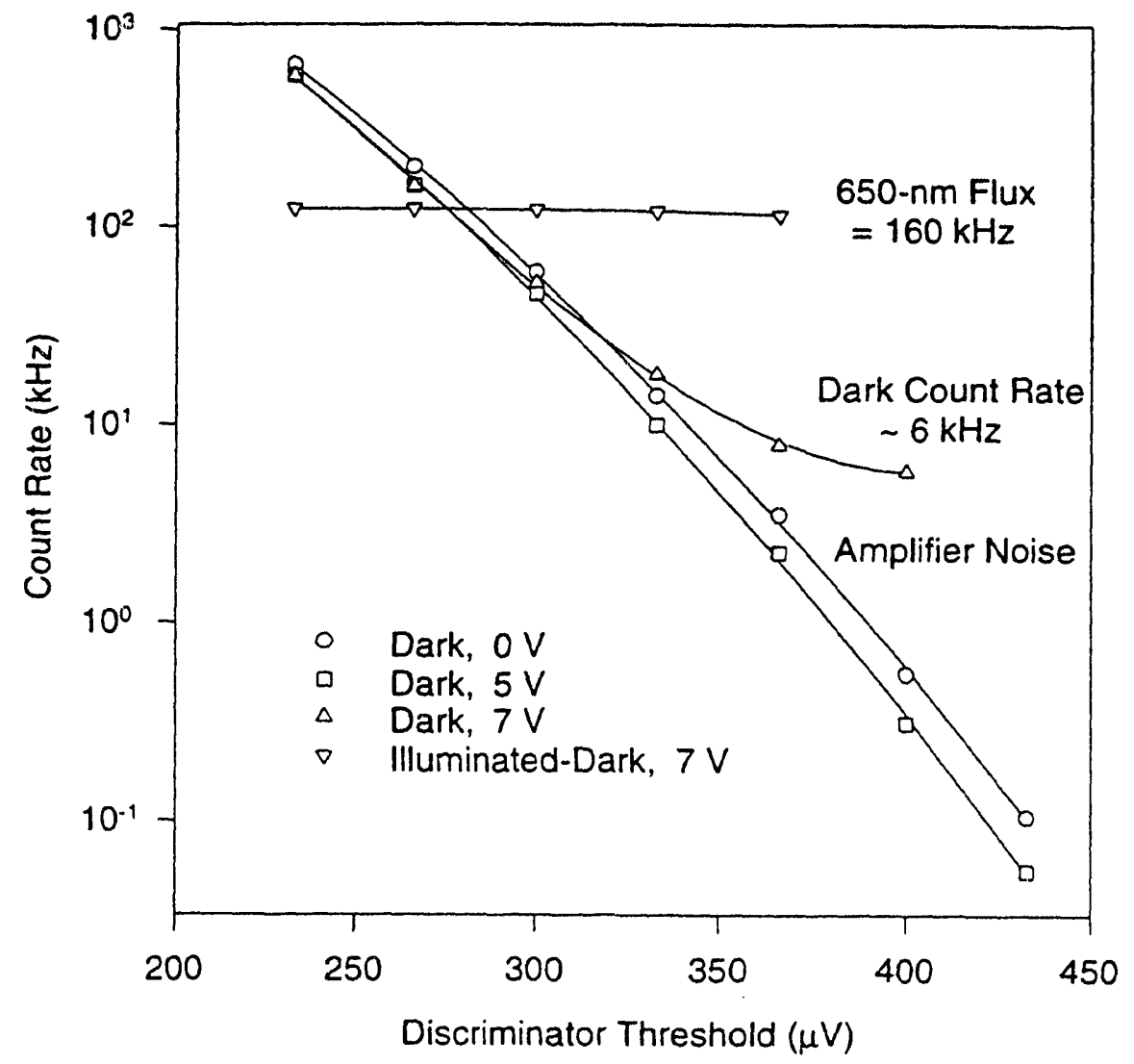

Figure 1."Measured count rate vs. discriminator threshold for different applied biases, with and without illumination"

The rate of background (all light blocked at the top of the fiber) was measured as a function of discriminator threshold for different bias voltages. The result is also shown on 
Fig. 1, in units of the charge integrator output. At bias voltages of 0 and $-5 \mathrm{~V}$, the spectrum is rapidly decreasing as a function of pulse height. This background has a gaussian shape of 90 microvolt standard deviation. Its shape and magnitude fit what is expected from an amplifier with input noise of $2 \mathrm{nV} / \mathrm{SQRT}(\mathrm{Hz})$ and the circuits time constants. At 0 and at $-5 \mathrm{~V}$, the curves are slightly different, as expected because the device capacitance depends on the bias voltage. At $-7 \mathrm{~V}$, the noise spectrum results from the superposition of the noise seen at 0 and $-5 \mathrm{~V}$, and of another noise far less dependant on discriminator level. The latter phenomenon may be due to electrons liberated in the device drift region by thermal agitation and to infra red radiation emitted at room temperature, transmitted down to the device via the fiber. The dependance of the latter background on discriminator level is expected to resemble the dependarce of the efficiency, shown on that same Fig. 1. At the discriminator level where most of the efficiency measurements were taken $(.37 \mathrm{mV})$, the total amount of background is in the range of 6000 to 8000 counts/sec, with a significant contribution of the noise component which we attribute to the amplifier.

Tests were also performed using a spherical mirror with the sphere center located at, the top of the fiber. The purpose was to catch photons reflected at various places in the apparatus and to reflect them back toward the device (giving them other chances to be counted). The efficiency was found to be increased to values in the range of 80 to $86 \%$, depending on the values set for the operating parameters. Sometimes, for two of the devices, consecutive measurements were taken with and without mirror, not changing any other experimental condition, just measuring the effect of the mirror. The mirror enhancement factor was systematically found to be $1.168+$ or -.01 for one device and 1.160 for the other.

Using the known index of refraction of the different materials on the path of the photons, one can compute the Fresnel reflections at all the interfaces. We can then estimate what happens to photons in the absence and in the presence of the spherical mirror. The mirror enhancement factor depends on the absorption in the fiber, which can therefore be determined. According to our measurements and this analysis, the internal iransmission factor (i.e. not counting reflections) is found to be $91+$ or $-3 \%$ in the fiber associated with one device, $90+$ or $-1.6 \%$ in the other. After the tests, the devices were dismounted and the absorption at $660 \mathrm{~nm}$ was measured directly. The internal transmission factor was $94.6 \%$ in the first fiber and $91 \%$ in the other, quite consistent with the values determined from the mirror enhancement factors. The transmission factor of virgin fibers was also measured and found consistent with the manufacture specifications, which correspond to a transmission of $99 \%$ in a fiber of the length used in our assembly, $19.0 \mathrm{~cm}$.

From the above analysis, we can conclude that the fibers have aged or have been damaged since they were manufactured and the amount of light absorbed has increased from $1 \%$ to about $8 \%$. The observed small degradation is ascribed to handling in assembly and to temperature cycling. The intrinsic efficiency of the silicon device with an optimum AR coating would be $96+$ or $-3 \%$, (taking into account a possible normalization error of our calibration apparatus estimated at $2 \%$ ).

\section{DISCUSSION}

We believe our results represent a significant breakthrough in visible light photon counting detectors. With the actual efficiency obtained in our test, $8.5 \%$. with a background of 
$7 \mathrm{kHz}$ as we measured it, and a timing accuracy of $10 \mathrm{~ns}$ (as determined in the test of last year), it sheild be possible to perform an EPR loophole free experiment if, in addition, the loss of one photon could be less than $3 \%$ and the visibility be $95 \%$ or better. However the margin of safety would be small. Therefore it is most important to look at possible improvements. Such improvements of the detector seem possible. Therefore EPR loophole free experiments are definitely in the realm of possibilities.

There are areas where improvements are possible

1. The plastic fiber could be replaced by a glass one if one can be found that absorbs infrared efficiently but does not age.

2. Each interface could be coated with a proper AR coating, to avoid the need of a spherical mirror. Timing accuracy would be improved too.

3. A cold amplifier could be used as a preamp to reduce amplifier noise.

4. A more sophisticated coincidence circuit (relying on zero crossing for instance) would improve timing accuracy.

The authors are indebted to $T$. Weber for his help in getting the measurements done and to Prof. R. Chiao for lending them equipment for the test.

\section{REFERENCES}

1. P.G. Kwiat, A.M. Steinberg, R.Y. Chiao, P.H. Eberhard, and M.D. Petroff, "Highefficiency single-photon detectors", Phys Rev A, 48, R867 (1993).

2. P.G. Kwiat, A.M. Steinberg, R.Y. Chiao, P.H. Eberhard, and M.D. Petroff, "Absolute efficiency and time-response measurement of single-photon detectors", Applied Optics. 38, 1844 (1994).

3. M.D. Petroff, M.G. Stapelbroek and W.A. Kleinhans, "Detection of individual 0.428 micrometer wavelength photons via impurity-impact ionization in a solid state photomultiplier," Appl. Phys. Lett. 51, 406-408 (1987).

4. A.W. Lightstone, A.D. MacGiregor, D.E. MacSween, R.J. McInty, C. Trottier, and P.P. Webb, "Photon counting modules using RC'A silicon avalanche photodiodes." Electron. Eng. 61, 37-47 (1989).

5. G.B. Turner, M.G. Stapelbroek, M.D. Petroff, E.W. Atkins. and H.H. Hogue, "Visible Light Photon Counters for Scintillating Fiber Applications: I. "Characteristics and Performance", Proceedings of the Workshop on Scintillating Fiber Detectors. Notre Dame Univ. October 24-28, 1993, Editor R. Ruchti, World Scientific Publishing Co Pte Ltd. 1994.

6. M.G. Stapelbrock and M.D. Petroff, "Visible Light Photon Counters for Scintillating Fiber Applications: II. Principles of Operation", Proceedings of the Workshop on Scintillating Fiber Detectors, Notre Dame Univ.,October 24-28, 1993, Editor R. Ruchti, World Scientific Publishing Co Pte Ltd. 1994. 

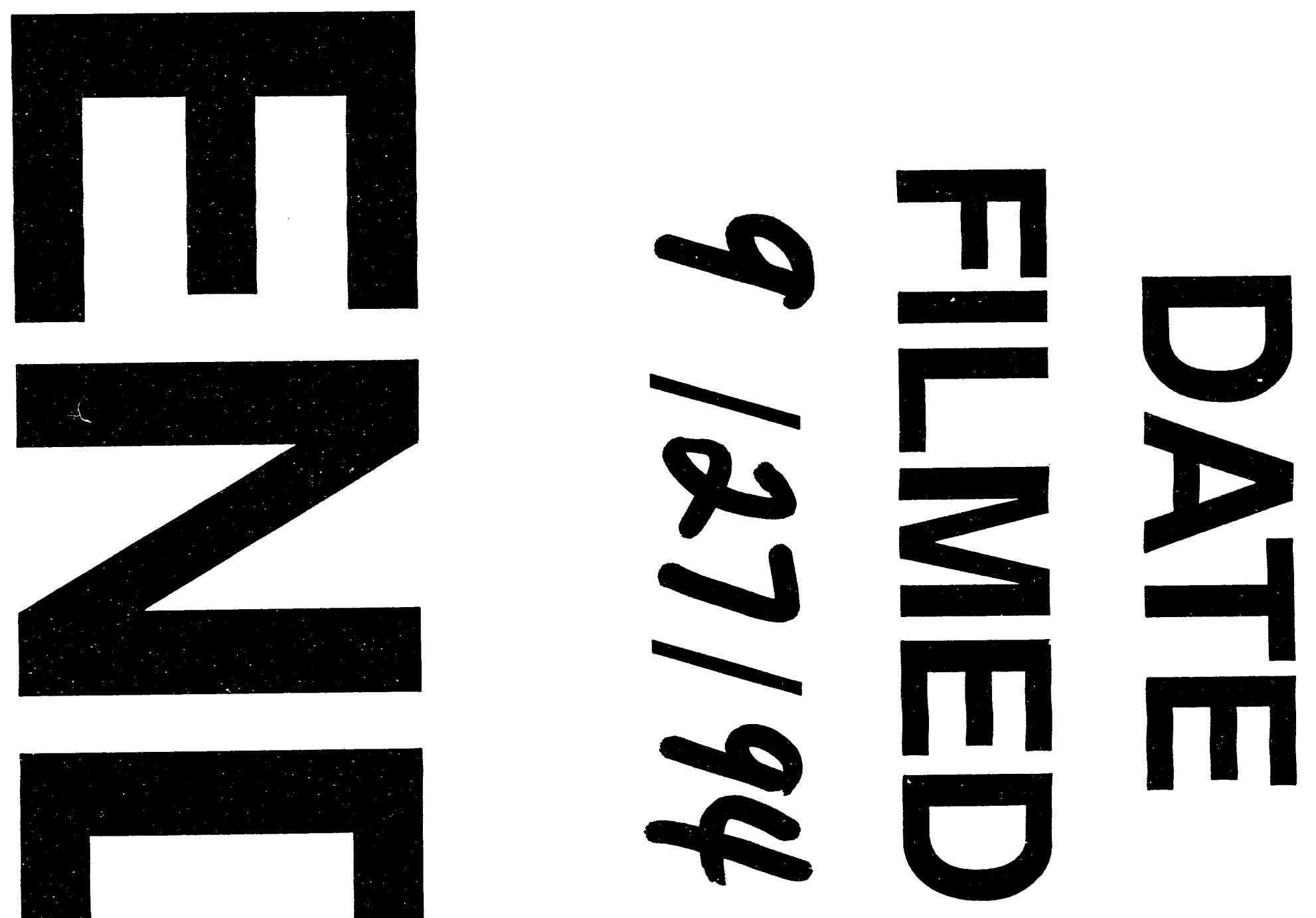
